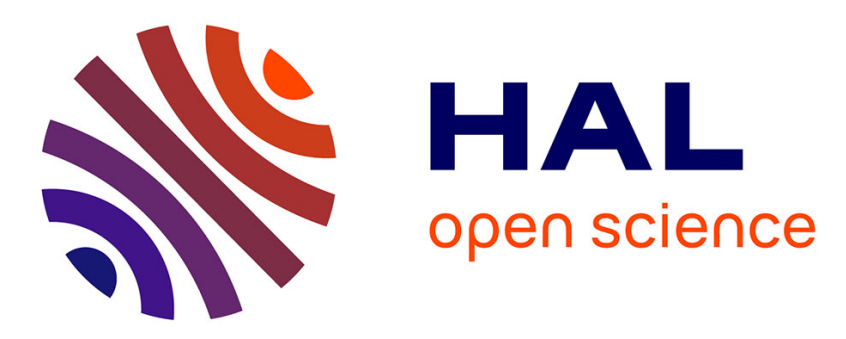

\title{
Droplets in Microchannels: Dynamical Properties of the Lubrication Film
}

\author{
Axel Huerre, Olivier Theodoly, Alexander Leshansky, Marie-Pierre Valignat, \\ Isabelle Cantat, Marie-Caroline Jullien
}

\section{> To cite this version:}

Axel Huerre, Olivier Theodoly, Alexander Leshansky, Marie-Pierre Valignat, Isabelle Cantat, et al.. Droplets in Microchannels: Dynamical Properties of the Lubrication Film. Physical Review Letters, 2015, 115 (6), pp.064501. 10.1103/PhysRevLett.115.064501 . hal-01201928

\section{HAL Id: hal-01201928}

\section{https://hal-univ-rennes1.archives-ouvertes.fr/hal-01201928}

Submitted on 23 Sep 2015

HAL is a multi-disciplinary open access archive for the deposit and dissemination of scientific research documents, whether they are published or not. The documents may come from teaching and research institutions in France or abroad, or from public or private research centers.
L'archive ouverte pluridisciplinaire HAL, est destinée au dépôt et à la diffusion de documents scientifiques de niveau recherche, publiés ou non, émanant des établissements d'enseignement et de recherche français ou étrangers, des laboratoires publics ou privés. 


\title{
Droplets in microchannels: dynamical properties of the lubrication film
}

\author{
Axel Huerre ${ }^{1}$, Olivier Theodoly ${ }^{2}$, Alexander Leshansky ${ }^{3}$, \\ Marie-Pierre Valignat ${ }^{2}$, Isabelle Cantat ${ }^{4}$, and Marie-Caroline Jullien ${ }^{1}$ \\ ${ }^{1} M M N$, UMR CNRS Gulliver 7083, PSL research University, \\ ESPCI ParisTech, 10 rue Vauquelin, F-75005 Paris, France \\ ${ }^{2}$ LAI, INSERM UMR_S 1067, CNRS UMR 7333, \\ Aix-Marseille Universite 13009 Marseille, France \\ ${ }^{3}$ Department of Chemical Engineering, Technion-IIT, Haifa, 32000, Israel \\ ${ }^{4}$ IPR, UMR CNRS 6251, Universite de Rennes 1, 35000 Rennes, France
}

(Dated: June 12, 2015)

\begin{abstract}
We study the motion of droplets in a confined, micrometric geometry, by focusing on the lubrication film between droplet and wall. When capillary forces dominate, the lubrication film thickness evolves non linearly with the capillary number due to viscous dissipation between meniscus and wall. However, this film may become thin enough (tens of nanometers) that intermolecular forces come into play and affect classical scalings. Our experiments yield highly resolved topographies of the shape of the interface and allow us to bring new insights into droplet dynamics in microfluidics. We report the novel characterization of two dynamical regimes as the capillary number increases: (i) at low capillary numbers, the film thickness is constant and set by the disjoinging pressure, while (ii) above a critical capillary number, the interface behavior is well described by a viscous scenario. At a high surfactant concentration, structural effects lead to the formation of patterns on the interface, which can be used to trace the interface velocity that yield direct confirmation of boundary condition in viscous regime.
\end{abstract}

The dynamics of a droplet confined between solid walls and pushed by a surrounding liquid is an old problem, however recent theories are still being developed to describe unexplored regimes and experimental characterizations are still lacking to shed light on these novel developments. A complete understanding of the droplet velocity calls for accurate knowledge of the dissipation mechanisms involved, particularly in the lubrication film. Our understanding of the lubrication properties of menisci travelling in confined geometries has been steadily refined since the pioneering work of Taylor \& Saffman [1]. Notably, the influence of the lubrication film left along the wall by the moving meniscus was first taken into account by Bretherton, who investigated the motion of an inviscid bubble in a cylindrical tube [2]. Far from the meniscus, this dynamical film reaches a uniform thickness $h_{\infty}$, related to the bubble velocity through the capillary number $C a=\mu_{f} U_{d} / \gamma$, where $U_{d}$ is the bubble velocity, $\mu_{f}$ the viscosity of the continuous phase, and $\gamma$ the surface tension. When the capillary pressure dominates over the viscous stress, i.e. in the regime where $C a \ll 1$, the thickness of the film follows $h_{\text {Breth }}=1.34 \mathrm{raa}^{2 / 3}$, where $r$ is the radius of the capillary tube. Besides bubbles, the case of viscous droplets remains however largely unexplored. A recent theoretical advance in the field by Hodges et al. [3] shows by numerical calculations of the whole flow pattern that significant corrections in the thickness of lubrication films can arise at very low $C a$. Furthermore, the regime of the Bretherton theory is only valid for lubrication films thicker than the molecular sizes or than the range of interfacial interactions. The typical velocities and lengthscales involved in droplet-based microfluidics would lead to lubrication films $h_{\infty} \sim 7$ -
$8 \mathrm{~nm}$ for oil-in-water droplets travelling at $100 \mu \mathrm{m} \cdot \mathrm{s}^{-1}$ in $15 \mu \mathrm{m}$-deep channels. This is below the thickness of the common black film (i.e. the equilibrium film balancing electrostatic repulsion and Van der Waals attraction, DLVO theory), which is of about $20 \mathrm{~nm}$ for an aqueous phase containing SDS $[4,5]$. Experimental investigations of dynamical properties of nanometric lubrication films, typically accessible at low $C a$, are largely lacking $[6,7]$.

Boundary conditions are of prime importance for the determination of the lubrication film thickness $[8,9]$. In this respect, three asymptotic regimes, corresponding to three boundary conditions at the interface, have been identified for bubbles: $i$ ) in the stress-free case, the fast remobilization of surfactants in the outer phase leads to a perfectly compressible interface, as it would be without surfactants; oppositely, ii) the sliding case corresponds to an incompressible interface with identical interface and bubble velocities as in a solid case, $U_{\mathrm{int}}=U_{d}$ in the laboratory frame; and iii) in the rolling case, $U_{\mathrm{int}}=0$ : the interface is incompressible in the film, it is created and suppressed at the front and rear of the bubble respectively. The scaling law is not affected by these different boundary conditions, but numerical prefactors are introduced in the expression of $h_{\infty}$, which becomes $h_{\text {Breth }}$, $2^{2 / 3} h_{\text {Breth }}$, or $4^{2 / 3} h_{\text {Breth }}$ respectively $[9,10]$. Moreover, the scaling law can be modified by the droplet viscosity. Considering classical lubrication approach, Teletzke et al. showed that there is a transition for the prefactor from 1 to $2^{2 / 3}$ for viscosity ratii about $10^{3}$ [6]. Nevertheless, Hodges et al. [3] took into account the whole non trivial flow pattern inside and outside the droplet to derive a thickness correction $\delta h_{\text {visc }}=h_{\infty}-h_{\text {Breth }}$ for a droplet with a viscosity contrast $\lambda=\mu_{d} / \mu_{f}=25$ which 
verifies $\delta h_{\text {visc }}=8.68 H C a$. Droplet viscosity thus leads to thicker films than the stress-free case in this scenario. In this letter, we propose an unprecedented experimental analysis of lubrication films at low capillary numbers for viscous oil in water droplets stabilized by surfactants. A quantitative optical measurement of lubrication film thickness as small as $20 \mathrm{~nm}$ and interface velocities allows us to shed new light on the low $C a$ regime, where both hydrodynamic and interfacial forces effects are expected to yield unexplored deviations from the Bretherton theory. We expect these results, which shed new light on droplet dynamics, to be of broad interest in the rapidly growing research field of droplet-based microfluidics [1114].

Oil-in-water droplets of radius $R=55 \pm 2 \mu \mathrm{m}$ are generated at a T-junction in a polydimethylsiloxane (PDMS - Sylgard 184) microfluidic system [15, 16], and enter a 2,500x9,000 $\mu \mathrm{m}$ Hele-Shaw cell of height $2 H=25 \pm 1$ $\mu \mathrm{m}$. The outlet is directly punched in the cell, to avoid a pressure drop from a channel outlet that might induce deformation of the high-aspect-ratio cavity. The droplets are mineral oil (SIGMA 8042-47-5) in deionized water with Sodium Dodecyl Sulfate (SDS) at 1.42 and 4.72 $\mathrm{g} / \mathrm{L}$ (respectively 0.6 and 2 times the CMC). The surface tension between the two fluids is $\gamma=1.6 \cdot 10^{-2} \mathrm{~N} / \mathrm{m}$ at $0.6 \mathrm{CMC}$ and $\gamma=1 \cdot 10^{-2} \mathrm{~N} / \mathrm{m}$ at $2 \mathrm{CMC}$. The viscosities are $\mu_{f}=10^{-3} \mathrm{~Pa} \cdot \mathrm{s}$ for the aqueous solution and $\mu_{d}=25 \cdot 10^{-3} \mathrm{~Pa} \cdot \mathrm{s}$ for the mineral oil, with a precision of $1 \cdot 10^{-4} \mathrm{~Pa} \cdot \mathrm{s}$. The oil droplets do not wet the walls of the cell so that there are no contact lines. We impose the water flow rate (Nemesys controller), ranging from 2 to $100 \mu \mathrm{L} / \mathrm{min}$. Corresponding droplet velocities vary between $50-5,000 \mu \mathrm{m} / \mathrm{s}$ and capillary numbers between $6 \cdot 10^{-6}$ and $5 \cdot 10^{-4}$. Lubrication film thicknesses are measured using Reflection Interface Contrast Microscopy (RICM) [17, 18]. The wavelength used is $550 \mathrm{~nm}$, so that two successive extrema in the interference pattern correspond to a film thickness difference about $130 \mathrm{~nm}$, and the optical indexes used in the model are 1.515, 1.33, 1.467 and 1.412 respectively for the glass plate, water solution, mineral oil and PDMS. The movies are acquired with a high speed camera (Photron Fastcam SA4) at a maximum frequency of $1000 \mathrm{~Hz}$, which results in a maximum of 6 blurred pixels at the highest velocities. The pixel resolution is $0.6 \mu \mathrm{m}$; the pictures are normalized by the background without droplet to get rid of spatial inhomogeneities in lighting. The thicknesses $h_{\infty}$ of the wetting film reported on Fig.2 and 3a are obtained by averaging the local thickness over a rectangular central zone where the film thickness is constant (see Fig.1b) and over 15 successive frames. The error bars result from the quadratic sum of the statistical error (from the 15 frames), the model error (from the numerical aperture) and the reference error in the normalized intensity calculation. Details about the RICM method and error bars calculation are given in the Supplementary Materials.

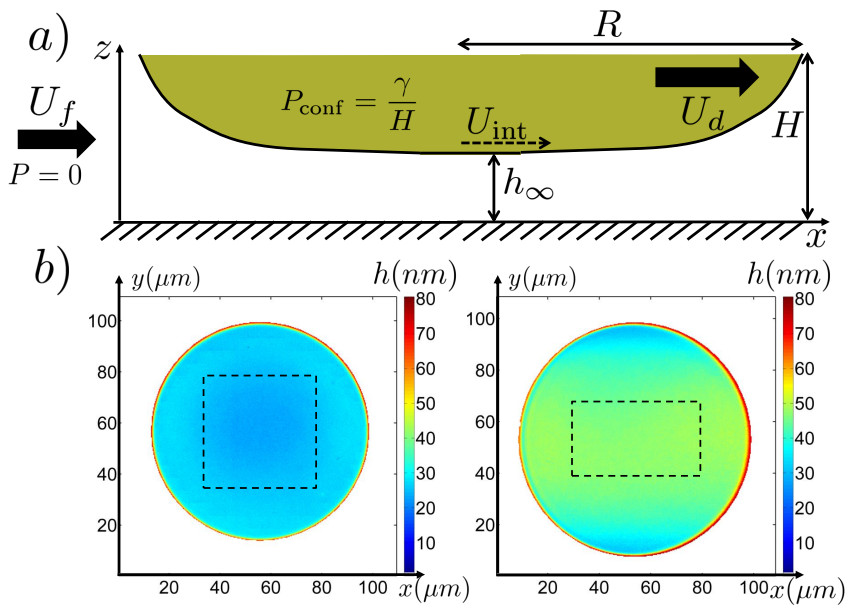

FIG. 1. a: Schematics of the experiment and notations used in the text. b: Left: plot of the film thickness for $\mathrm{Ca}=$ $3.1 \cdot 10^{-6}$. Right: plot of the film thickness for $C a=8.1 \cdot 10^{-5}$. Dotted rectangles: averaging area for the mean film thickness $h_{\infty}$.

The first set of experiments was performed below the CMC (0.6 CMC i.e. $4.92 \mathrm{mM}$ ). Schematics and experimental topographies are shown in Fig.1 to illustrate the geometry of the droplets. In particular, plots of the lubrication film thickness are given for two droplets flowing at different velocities, see Fig.1b (the full 3D profiles are represented as videos in the Supplementary Materials). As the droplet velocity increases above $C a \sim 7 \cdot 10^{-5}$, the shape of the interface smoothly changes from a homogeneous film of constant thickness $\left(\mathrm{Ca}<7 \cdot 10^{-5}\right.$, Fig.1b. left) to a more complex $3 \mathrm{D}$ shape, while the film thickness at the center increases $\left(\mathrm{Ca}>7 \cdot 10^{-5}\right.$, Fig.1b. right). Note that to our knowledge, no model so far has predicted the 3D shape of a flowing droplet in a Hele-Shaw cell: though the full characterization of these profiles is not the aim of this paper, our results give a first picture of these complex shapes.

Measuring the film thickness $h_{\infty}$ allows us to quantify the transition between two dynamical regimes as the capillary number increases, see Fig.2. Above a critical capillary number $C a^{*} \sim 3 \cdot 10^{-5}$, the film thickness increases with the capillary number according to the Hodges et al. [3] theory, while below $C a^{*}$ it remains constant, in agreement with theoretical predictions by Teletzke et al. [6]. Indeed, at high velocities, the film thickness is governed by a balance between viscous entrainment and capillary suction; the film is thick enough for short range interactions to be negligible. At low velocities, the viscous entrainment is reduced, which results in thinner films where short range repulsive effects, represented by the disjoining pressure $\Pi_{\mathrm{disj}}$, become dominant.

To further characterize the capillary regime, $\mathrm{Ca}>$ $C a^{*}$, we compare our data to theoretical predictions for different conditions at the interface: the stress-free case, 


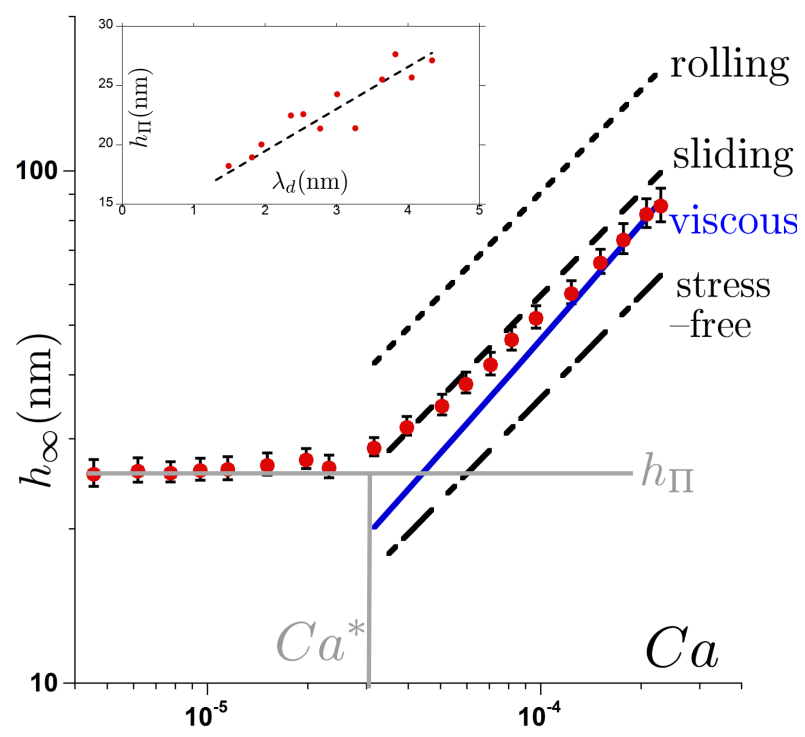

FIG. 2. Film thickness as a function of the capillary number. $(\bullet)$ : experimental data. Lines : theoretical predictions for different boundary conditions[10]. - - - : stress-free; - - : sliding; - - : rolling. - : stress continuity interface with a viscous drop $\left(\mu_{d} / \mu_{f}=25\right)[3] . h_{\Pi}=$ static film thickness. Inset: static film thickness $h_{\Pi}$ as a function of the Debye length $\lambda_{D}$.

the sliding case $\left(U_{\mathrm{int}}=U_{d}\right.$ in the laboratory frame), the rolling case $\left(U_{\mathrm{int}}=0\right)[9,10]$, and the viscous model: $\mu_{f} \partial u / \partial z=\mu_{d} \partial v / \partial z$ [3]. As seen above, these different conditions affect the numerical prefactor to Bretherton's scaling $h_{\infty} \propto \mathrm{HCa}^{2 / 3}$ for the first three boundary conditions, while for the viscous model there is an additionnal linear scaling in $C a$. These predictions are plotted on Fig.2 with no adjustable parameter, showing a quantitative agreement between our data and the theoretical curve for the viscous model $h_{\mathrm{visc}}=h_{\mathrm{Breth}}+\delta h_{\mathrm{visc}}=$ $1.34 \mathrm{HCa}^{2 / 3}+8.68 \mathrm{HCa}$ (see Supplementary Materials) and the sliding model. We will show in the second part of the paper that the interface velocity is negligible compared to the droplet viscosity allowing us to finally discard the sliding regime. While sliding or stress-free motions have been previously reported [19], to our knowledge this is the first experimental observation of a viscous regime for droplets.

When the capillary number decreases below $C a^{*}$, disjoining pressure effects become dominant and the lubrication film thickness reaches a plateau. This asymptotic value is consistent with the static film thickness $h_{\Pi} \sim 25 \mathrm{~nm}$ measured for a droplet at rest $\left(U_{d}=0\right)$, which is set by the disjoining pressure $\gamma / H$ (i.e. imposed by the confinement). In these experimental conditions, the disjoining pressure is governed by electrostatic effects rather than Van der Waals or steric forces (common black film[20]; see the thick branch on the disjoining pressure isotherm in Supplementary Materials). This is verified by

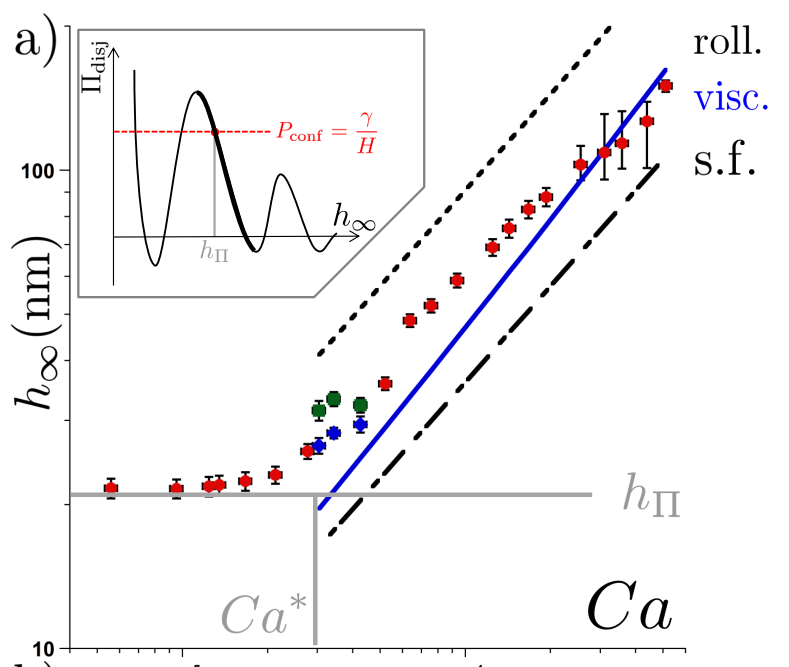

b)

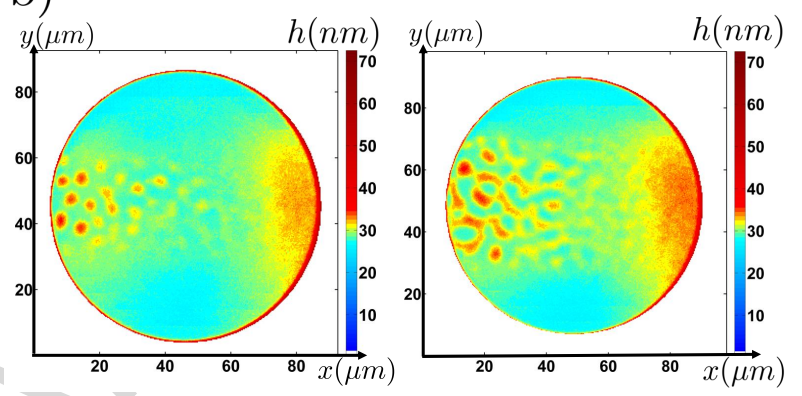

FIG. 3. a: Local film thickness as a function of the capillary number. (•): Experimental data. For $3 \cdot 10^{-5}<C a<5 \cdot 10^{-5}$, two thicknesses coexist: $(\square) h_{\max }$ and $(\downarrow) h_{\min }$. Lines: see Fig.2. Inset: sketch of the oscillatory disjoining pressure isotherm, according to a structural model [20]. The bold line corresponds to the common black film. b: spatial plot of the film thickness, for two capillary numbers in the transition zone (left: $C a=3 \cdot 10^{-5}$; right: $C a=3.4 \cdot 10^{-5}$ ). $h_{\max }$ is coded in red and $h_{\min }$ in light blue. Note that the color scales are adjusted in order to enhance the pattern contrast.

the linear relation between the static film thickness $h_{\Pi}$ and the Debye length $\lambda_{D}=0.3 / \sqrt{C_{\mathrm{SDS}}(\mathrm{mol} / \mathrm{L})}$, Fig.2 (inset). The Debye length was tuned down by adding $\mathrm{NaCl}$ up to $0.4 \mathrm{M}$, while the SDS concentration was adjusted to maintain 0.6 CMC [21].

Having investigated the electrostatic contribution of the disjoining pressure, experiments above the $\mathrm{CMC}$ were performed to explore the influence of structural effects. At 2 CMC, the evolution of the film thickness with $\mathrm{Ca}$ is qualitatively similar, but the plateau value below $C a^{*}$ is lower: $h_{\Pi} \sim 20 \mathrm{~nm}$, see Fig.3a. Indeed, increasing the SDS concentration decreases $\lambda_{D}$, and thus the equilibrium film thickness.

Another difference from the low surfactant concentration results is the coexistence of two distinct thicknesses, $h_{\min }$ and $h_{\max }$, at the transition between the two regimes (Fig.3a).

The patterns visible on Fig.3b are characteristic of a 


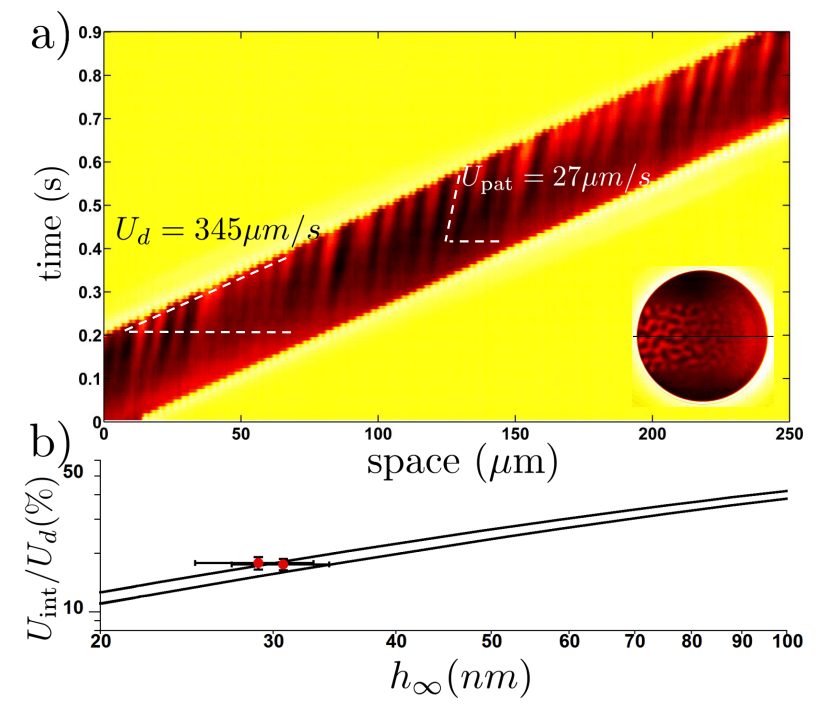

FIG. 4. a: Spatio-temporal plot of a droplet cross section (raw interference patterns). The section is taken along the direction of movement, as shown in the inset. Brighter colors correspond to higher thicknesses, in arbitrary units. The droplet and pattern velocities are measured from the angles between the dashed white lines: $U_{d}=345 \mu \mathrm{m} / \mathrm{s}$ and $U_{\text {pat }}=27 \mu \mathrm{m} / \mathrm{s}$. b: Normalized interface velocity, $\left(U_{\text {int }} / U_{d}\right) * 100$, as a function of the lubrication film thickness $h_{\infty}$. $(\bullet)$ : Experimental data. - : upper and lower estimations assuming a viscous model.

spinodal decomposition, sometimes involved in disjoining pressure-governed phenomena $[4,22]$. In micellar solutions, such a phenomenon is due to the oscillatory shape of the disjoining pressure isotherm, see inset in Fig.3a $[4,5,20]$. These oscillations stem from structural interactions between highly concentrated micelles, and have a wavelength equal to the effective micelle size, i.e. the diameter of a micelle plus twice the Debye length. Here, the difference $h_{\max }-h_{\min } \sim 6 \mathrm{~nm}$ indeed corresponds to the size of a spherical SDS micelle and its water environment $\left(r_{\text {mic }} \sim 2 \mathrm{~nm}, \lambda_{D}=2.3 \mathrm{~nm}\right)[5]$, and is therefore consistent with a transition taking place between two successive branches of the isotherm.

These patterns can also be used to estimate the velocity of the interface. A spatio-temporal plot of the lubrication film thickness taken along a cross-section of the droplet in the direction of motion is shown in Fig.4.a. Such a picture gives access to the droplet velocity $U_{d}$, deduced from the slope of the droplet edge, and to the pattern velocity $U_{\text {pat }}$. The film thickness fluctuations $h(x, t)$ are related to the interface velocity through the lubrication equations, leading to $U_{\text {pat }}=U_{\text {int }} / 2$ (see Supplementary Materials for detailed calculation). We measured $U_{\text {pat }}=27 \mu \mathrm{m} \cdot \mathrm{s}^{-1}$, i.e. $U_{\mathrm{int}}=54 \mu \mathrm{m} \cdot \mathrm{s}^{-1}$ : the interface velocity is thus much smaller than the droplet velocity $U_{d}=345 \mu \mathrm{m} . \mathrm{s}^{-1}$, allowing us to disregard a sliding scenario.
We can now deduce the surface tension profile along the interface over the whole wetting film from the droplet and interface velocities. The stress balance at the interface writes: $\partial \gamma / \partial x=\mu_{f} \partial u / \partial z-\mu_{d} \partial v / \partial z$. The normal velocity derivatives are taken from a parabolic profile inside the droplet and a simple shear profile in the film, assuming $h_{\infty} \ll H$. The stress balance is integrated over the film length $2(R-H)$ from the front to the rear of the droplet. This leads to $\Delta \gamma_{\text {film }}=$ $\left[\mu_{f} U_{\mathrm{int}} / h_{\infty}-3 \mu_{d}\left(U_{d}-U_{\mathrm{int}}\right) / H\right] \times 2(R-H)$, which we solve numerically to find a surface tension difference of $4 \cdot 10^{-6} \pm 3 \cdot 10^{-5} \mathrm{~N} / \mathrm{m}$ for $C a=3.45 \cdot 10^{-5}$. This very low value allows us to disregard the influence of surface tension gradients on the dynamics. This confirms that the model for two immiscible pure liquids [3] provides a relevant description for our experiment, despite the presence of surfactants in the solution. Assuming this remains true over the whole range of capillary numbers, we can then predict the value of the interfacial velocity: $U_{\text {int }} / U_{d}=1 /\left(1+H /\left(3 \lambda h_{\infty}\right)\right)$. The upper and lower estimations (considering errors on $\lambda$ and $H$ ) are plotted in figure $4 \mathrm{~b}$. The experimental points for two different capillary numbers at the transition (where patterns are seen) are also plotted and lie between the two theoretical curves. Such quantitative data could prove useful to rationalize the complex behavior of the interface, though this would go beyond the scope of this paper.

At high confinement, two regimes have been evidenced in the dynamical properties of the lubricatin film between a traveling droplet and a solid wall. Two distinct regimes are evidenced: a capillary regime at large capillary numbers, as classically reported, and a regime governed by the disjoining pressure at lower capillary numbers. We expect this first experimental characterization of the disjoining pressure regime to open the way towards further work, such as the static or dynamical study of the disjoining pressure in a liquid-liquid-solid configuration. Two models have been discussed and characterized showing the relevance of Teletzke et al. model in the disjoining regime [6] and the relevance of Hodges et al. model [3] in the capillary regime; the latter emphasizing the effect of droplet viscosity, even at low viscosity ratio. These observations are of prime importance for the community and should impact research focused more generally on diphasic flows at the microscales. Moreover, measuring the complete topography of the lubrication film uncovered new fundamental information: at high capillary numbers, the lubrication film adopts a complex catamaran-like shape (Supplementary Materials, movie), which should prove of interest for 3D modeling. For intermediate capillary numbers, above the $\mathrm{CMC}$, a spinodal decomposition gives access to interfacial properties that evidence a complex viscous behavior; these experiments shall pave the way towards the study of more complex situations, such as the influence of non soluble surfactants and its competition with viscosity. 


\section{ACKNOWLEDGEMENTS}

This work was supported by CNRS, IPGG (Equipex ANR-10-EQPX-34), ESPCI, ANR under the contract 13BS09-0011-01 and INSERM. A. M. L. acknowledges the support of the Israel Science Foundation (ISF) via the grant \#1319/09 and Joliot Chair visiting position at Ecole Supérieure de Physique et Chimie de Paris (ESPCI). We acknowledge valuable discussions with Michael Schindler and Cécile Monteux.

[1] G. Taylor and P. G. Saffman, Q J Mechanics Appl Math 12, 265 (1959).

[2] F. Bretherton, J. Fluid Mech. 10, 166 (1961).

[3] S. R. Hodges, O. E. Jensen, and J. M. Rallison, J. Fluid Mech. 501, 279 (2004).

[4] A. Nikolov and D. Wasan, J. Colloid Interface Sci. 133, 1 (1989).

[5] V. Bergeron and C. J. Radke, Langmuir 8, 3020 (1992).

[6] G. F. Teletzke, H. T. Davis, and L. Scriven, Rev. Phys. App. 23, 989 (1988).

[7] J.-D. Chen, J. Colloid Interface Sci. 109, 341 (1986).
[8] A. Huerre, V. Miralles, and M.-C. Jullien, Soft Matter 10, 6888 (2014).

[9] I. Cantat, Phys. Fluids 25, 031303 (2013).

[10] A. Q. Shen, B. Gleason, G. H. McKinley, and H. A. Stone, Phys. Fluids 14, 4055 (2002).

[11] C. N. Baroud, F. Gallaire, and R. Dangla, Lab Chip 10, 2032 (2010).

[12] N. Champagne, R. Vasseur, A. Montourcy, and D. Bartolo, Phys. Rev. Lett. 105, 044502 (2010).

[13] I. Shani, T. Beatus, R. H. Bar-Ziv, and T. Tlusty, Nature Phys. 10, 140 (2014).

[14] H. A. Stone and S. Thutupalli, Nature Phys. 10, 87 (2014).

[15] Y. Xia and G. M. Whitesides, Annu. Rev. Mater. Sci. 28, 153 (1998).

[16] V. van Steijn, C. R. Kleijn, and M. T. Kreutzer, Phys. Rev. Lett. 103, 214501 (2009).

[17] A. S. G. Curtis, J. Cell Biol. 20, 199 (1964).

[18] O. Theodoly, Z.-H. Huang, and M.-P. Valignat, Langmuir 26, 1940 (2010).

[19] N. D. Denkov, S. Tcholakova, K. Golemanov, V. Subramanian, and A. Lips, Colloids Surf., A 282, 329 (2006).

[20] V. Bergeron, J. Phys.: Condens. Matter 11, R215 (1999).

[21] R. J. Williams, J. N. Phillips, and K. J. Mysels, Trans. Faraday Soc. 51, 728 (1955).

[22] M. L. Pollard and C. J. Radke, J. Chem. Phys 101, 6979 (1994). 